\title{
Simple descriptors for proton-conducting perovskites from density functional theory
}

Bork, Nicolai Christian; Bonanos, Nikolaos; Rossmeisl, Jan; Vegge, Tejs

Published in:

Physical Review B Condensed Matter

Link to article, DOI:

10.1103/PhysRevB.82.014103

Publication date:

2010

Document Version

Publisher's PDF, also known as Version of record

Link back to DTU Orbit

Citation (APA):

Bork, N. C., Bonanos, N., Rossmeisl, J., \& Vegge, T. (2010). Simple descriptors for proton-conducting perovskites from density functional theory. Physical Review B Condensed Matter, 82(1), 014103. https://doi.org/10.1103/PhysRevB.82.014103

\section{General rights}

Copyright and moral rights for the publications made accessible in the public portal are retained by the authors and/or other copyright owners and it is a condition of accessing publications that users recognise and abide by the legal requirements associated with these rights.

- Users may download and print one copy of any publication from the public portal for the purpose of private study or research.

- You may not further distribute the material or use it for any profit-making activity or commercial gain

- You may freely distribute the URL identifying the publication in the public portal 


\title{
Simple descriptors for proton-conducting perovskites from density functional theory
}

\author{
N. Bork and N. Bonanos \\ Fuel Cells and Solid State Chemistry Division, Ris $\phi$ National Laboratory for Sustainable Energy, Technical University of Denmark, \\ DK-4000 Roskilde, Denmark \\ J. Rossmeisl \\ Center for Atomic-scale Materials Design, Department of Physics, Technical University of Denmark, \\ DK-2800 Kongens Lyngby, Denmark \\ T. Vegge* \\ Materials Research Division, Risф National Laboratory for Sustainable Energy, Technical University of Denmark, \\ DK-4000 Roskilde, Denmark
}

(Received 1 October 2009; revised manuscript received 29 April 2010; published 8 July 2010)

\begin{abstract}
A series of (pseudo)cubic perovskites, $\mathrm{ABO}_{3}$, have been investigated using density functional theory calculations. The structures have been optimized and thermodynamic properties and activation energies for the relevant steps of the hydrogen/proton diffusion mechanism have been calculated using the nudged elastic band path technique. We find a strong correlation between the $\mathrm{O}-\mathrm{H}$ binding energy for hydrogen/proton uptake in perovskites and the energy barriers involved in the observed Grotthuss-type diffusion process. We demonstrate the possibility of estimating diffusion rates based on $\mathrm{O}-\mathrm{H}$ binding energy and temperature only, without determining transition states and vibrational frequencies. We determine the binding energy providing the optimal tradeoff between occupation and diffusion rate at a given temperature, and finally we show how these correlations can be used to suggest candidate materials with improved kinetic properties for potential application as hydrogen permeable membranes and proton-conducting electrolytes.
\end{abstract}

DOI: 10.1103/PhysRevB.82.014103

PACS number(s): 66.30.jp, 71.15.Mb, 82.20.Db

\section{INTRODUCTION}

The recent revival of interest in renewable energy has led to increased interest in energy technologies based on hydrogen and fuel cells. Contaminants in the hydrogen gas is an issue that needs to be dealt with since these degrade both lifetime and performance of fuel cells by active site poisoning. ${ }^{1}$ One way of purifying the feed gas is by diffusion through a dense hydrogen separation membrane hereby producing hydrogen virtually free of contaminants. Membranes may also be applied in the separation of $\mathrm{CO}_{2}$ and $\mathrm{H}_{2}$ synthesized from natural gas and potentially also as electrochemical sensors.

Typical membranes applied today are based on alloys of, e.g., palladium, silver, or niobium but oxide-based ceramics, e.g., perovskites $\left(\mathrm{ABO}_{3}\right)$, also exhibit hydrogen/proton permeability and offer potential savings compared to typical metallic membranes.

The main obstacle for perovskite based membranes is the low diffusivity of hydrogen in any pure perovskite. It has been found that doping the perovskite with $5-25 \%$ of a lower valence element, such as yttrium or neodymium, on the $B$ site can increase the concentration of protons in the membrane significantly and increase the hydrogen flux. ${ }^{2}$ Even though protons in perovskites have been studied extensively, there are still considerable uncertainties and the optimal membrane composition is yet to be determined. A complete investigation of all possible combinations of perovskite, dopant and dopant concentration is however practically impossible. To address this issue, we have searched for possible correlations between hydrogen flux, protonic concentration in the material and structural proper- ties, in order to find simple "descriptors" which can facilitate a more efficient screening of candidate materials. ${ }^{3}$ Similar investigations have previously been performed but no decisive breakthrough has been made.

Ranløv et al. ${ }^{4}$ have investigated $\mathrm{LnAl}_{0.95} \mathrm{Mn}_{0.05} \mathrm{O}_{3-y}$, $\mathrm{Ln}=(\mathrm{Y}$ and lanthanides$)$ and found that the ionic radius of the $\mathrm{Ln}$ ion correlates with several structural and thermodynamic properties of the material. Mitsui et al. ${ }^{5}$ were able to link kinetic properties for proton diffusion in perovskites to structural parameters, e.g., lattice constants. The existence of a strong correlation between prefactors and activation barriers has also been shown in the hydrogenation and dehydrogenation processes of various metals and metal hydrides. ${ }^{6}$

Grain boundaries, defects, and dopants create irregularities in the lattice which increase the complexity of both the thermodynamics and kinetics of the system. ${ }^{7}$ We have therefore undertaken a theoretical study of hydrogen/proton (henceforth referred to as proton) diffusion in the undoped pseudocubic and cubic perovskites listed in Table I. ${ }^{8}$ The complexity of the diffusion mechanism is thereby reduced due to the high-symmetry inherent in the investigated materials. In oxides and (pseudo)cubic perovskites, in particular, the possible diffusion paths are limited since the proton in all studied systems binds strongly to the lattice oxygen as shown in Fig. 1. We present results for the Grotthuss diffusional mechanism for individual protons where the host lattice distorts due to the $\mathrm{H} / \mathrm{OH}$ defect but remains unchanged with respect to bonding at any time during the diffusion. This is also referred to as "free proton migration" and is considered the primary mechanism for protonic transport in oxides. ${ }^{19,20}$ Only two distinct diffusional steps are required, namely, the transfer of a proton from one oxygen atom to another, termed 
TABLE I. Calculated cubic unit-cell volumes compared to experimental data. $\dagger$ indicates that an orthorhombic structure is preferred at room temperature. $\Delta E_{\mathrm{OH}}$ form is the formation energy of a hydrogen defect from reaction (2).

\begin{tabular}{lccc}
\hline \hline & \multicolumn{2}{c}{ Lattice volume $\left(\AA^{3}\right)$} & \multicolumn{1}{c}{$\Delta E_{\mathrm{OH} \text { form }}$} \\
\cline { 2 - 3 } Perovskite & Calc. & Expt. & $(\mathrm{eV})$ \\
\hline $\mathrm{BaTiO}_{3}$ & 62.1 & $63.5^{9}$ & 1.40 \\
$\mathrm{BaZrO}_{3}$ & 73.6 & $73.0^{10}$ & 2.15 \\
$\mathrm{BaNbO}_{3}$ & 67.4 & $65.9^{11}$ & 2.14 \\
$\mathrm{CaMnO}_{3}$ & 51.1 & $51.9^{12} \dagger$ & -1.92 \\
$\mathrm{CaTiO}_{3}$ & 57.5 & $53.6^{13} \dagger$ & -1.32 \\
$\mathrm{CaZrO}_{3}$ & 69.4 & $64.5^{14} \dagger$ & -2.66 \\
$\mathrm{PbTiO}_{3}$ & 62.1 & $62.6^{15}$ & 1.32 \\
$\mathrm{PbZrO}_{3}$ & 72.0 & $73.0^{15}$ & 1.61 \\
$\mathrm{SrNbO}_{3}$ & 65.5 & $65.5^{16}$ & 1.92 \\
$\mathrm{SrTiO}_{3}$ & 59.8 & $59.3^{17}$ & 1.40 \\
$\mathrm{SrZrO}_{3}$ & 72.0 & $71.5^{18}$ & 0.69 \\
\hline \hline
\end{tabular}

"H jump," and the rotation of the $\mathrm{OH}$ bond around the oxygen atom termed "OH rot." These steps are sufficient to describe the macroscopic protonic transport.

In the theoretical approach described here, we are able to treat each diffusional process separately and deduce information of the individual processes at the atomic scale, in contrast to an experimental approach where data originating from, e.g., impedance spectroscopy or the electromotive force often reflect an average over several mechanisms and effects. $^{21}$ Atomic details can be extracted from neutronscattering experiments ${ }^{22,23}$ but the high cost of these, combined with time consuming data analysis, makes this technique unsuitable for screening purposes.

\section{METHODS AND COMPUTATIONAL DETAILS}

The electronic-structure calculations have been performed using density functional theory (DFT) implemented in the DACAPO package ${ }^{24}$ developed at CAMD at the Technical University of Denmark. The exchange-correlation functional used is PW91. ${ }^{25}$ The electronic wave functions are expanded in plane wave functions with a cutoff energy of $350 \mathrm{eV}$ while the electronic-density energy cutoff was $550 \mathrm{eV}$. The ion cores are described by ultrasoft pseudopotentials. ${ }^{26}$

Periodic boundary conditions have been used in all calculations on $(2 \times 2 \times 2)$ perovskite supercells of approximately $(8 \AA)^{3}$ containing 40 atoms. The Brillouin zone has been sampled by a $(2 \times 2 \times 2)$ Monkhorst-Pack grid. ${ }^{27}$ Convergence is reached when the maximum force acting on any atom drops below $0.05 \mathrm{eV} / \AA$ and the optimization is performed using the quasi-Newton algorithm.

The minimum-energy paths and energy barriers have been determined using the nudged elastic band (NEB) method. ${ }^{28,29}$ Convergence criteria for the transition states are, as for optimizations, $0.05 \mathrm{eV} / \AA$.

Rate constants are calculated using transition state theory in the harmonic approximation (hTST). ${ }^{30}$ The pre-

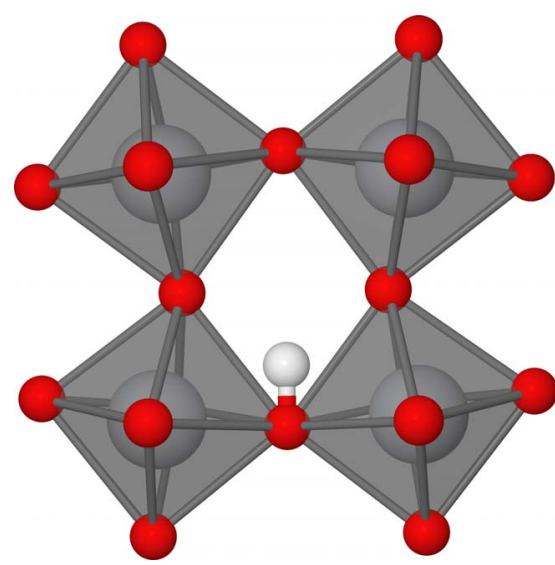

FIG. 1. (Color online) Conformation of most stable configuration of a hydrogenated $\mathrm{SrTiO}_{3}$ supercell. [Sr: omitted for clarity, Ti: gray (large), $\mathrm{O}$ : red, and $\mathrm{H}$ : white.]

exponential is, in the harmonic approximation, given as the ratio between the products of vibrational frequencies of the initial state, $\nu_{\text {initial }}$, and the vibrational frequencies of transition state, $\nu_{T S} \cdot{ }^{31,32}$ The reaction rate is thus given as

$$
k(T)=\frac{\prod \nu_{\text {initial }}}{\prod \nu_{T S}^{\ddagger}} \times \exp \left(\frac{-E_{a}}{k_{B} T}\right)
$$

where $\$$ indicates that the imaginary frequency is left out of the equation, resulting in unit of $\mathrm{s}^{-1}$. The vibrational frequencies of both optimized and transition states are calculated using a finite-difference approximation with displacements of $0.01 \AA$.

\section{RESULTS AND DISCUSSION}

The initial unit-cell optimization was performed while enforcing cubic $P 4 \mathrm{~mm}$ symmetry. The optimized cell volumes were in good agreement with experimentally obtained data, given the simplified model (see Table I). The largest deviation in lattice volumes were found in the cases of $\mathrm{BaNbO}_{3}$, $\mathrm{CaTiO}_{3}$, and $\mathrm{CaZrO}_{3}$ with deviations of $2.3 \%, 7.3 \%$, and $7.6 \%$, respectively. It should be noted that $\mathrm{CaTiO}_{3}$ and $\mathrm{CaZrO}_{3}$ are found experimentally to prefer an orthorhombic structure over the cubic in the relevant temperature range. ${ }^{12,13}$ All other deviations were below $2 \%$. Since not all structures are perfectly cubic, the structures were internally relaxed in the optimized cubic supercells to minimize any structural inaccuracies from subsequent calculations. These reoptimized structures were used as reference states.

A hydrogen atom was then introduced in the supercell and all internal degrees of freedom were again optimized. Using a Bader charge analysis, ${ }^{33}$ we found the local charge distribution on the proton almost identical irrespective of whether a proton or a neutral hydrogen atom is inserted. The hydrogen/proton is in both cases best described as $\mathrm{H}^{+0.5}, 34$ and the neutral hydrogen atom was therefore chosen for all further calculations to utilize the more accurately determined energies from neutrally charged systems. The $\mathrm{O}-\mathrm{H}$ distance was in all systems very close to $1.0 \AA$ and the $\mathrm{O}-\mathrm{H}$ bond 


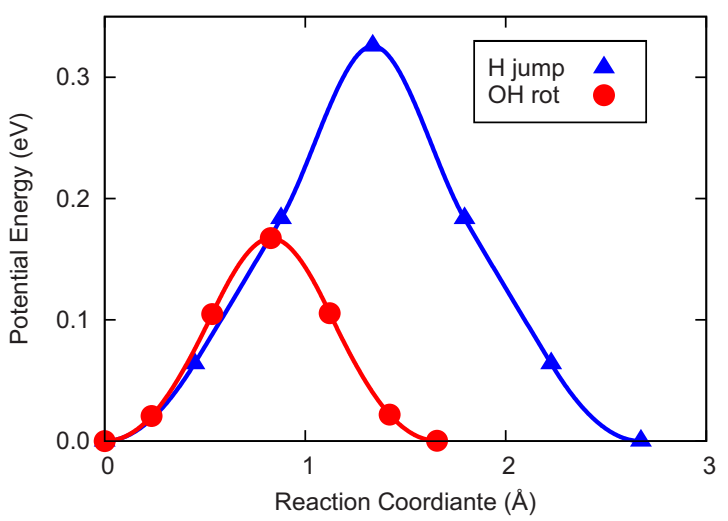

FIG. 2. (Color online) Illustration of potential energy surfaces of the minimum-energy paths linking reactant and product in the Grotthuss processes in $\mathrm{SrTiO}_{3}$. Markers indicate the configurations included in the actual NEB calculations.

was in all systems perpendicular to the B-B line and pointing between the $A$ cations (see Fig. 1) consistent with many previous studies, e.g., Björketun ${ }^{35}$ The insertion of the hydrogen atom into the supercell correspond to the reaction

$$
1 / 2 \mathrm{H}_{2}(g)+\mathrm{O}_{\mathrm{O}}^{x} \rightarrow \mathrm{OH}_{\mathrm{O}}^{a \bullet}+a e^{\prime}, \quad 0 \leq a \leq 1
$$

which is the first reaction of the hydrogen transport process, here in Kröger-Vink notation. $\mathrm{O}_{\mathrm{O}}^{x}$ denotes a structural oxygen with the " $x$ " indicating neutral charge compared to normal structural oxygen. $\mathrm{OH}_{\mathrm{O}}^{a \bullet}$ denote a hydroxyl defect on an oxygen site with charge " $a$ " compared to $\mathrm{O}_{\mathrm{O}}^{x}$ and $e^{\prime}$ denotes an electron. The free-energy differences associated with this reaction will be denoted $\Delta E_{\mathrm{OH}}$ form and are listed in Table I.

Having located the optimal proton positions in the lattice, we were able to identify reaction pathways using the NEB method (see Sec. II) for both of the Grotthuss processes. The reaction paths were in most cases symmetrical around the transition state as demonstrated in Figs. 2 and 3. The values of the activation barriers are listed in Table II.

In order to determine the diffusional rates using hTST, we also calculated the vibrational frequencies for the optimized structures and the transition states. Since an accurate determination of vibrational frequencies is a comparatively demanding task in terms of computational expense only the atoms closest to the proton were free to move while the rest of the atoms were fixed in space. We found that including the four closest $A$ ions, the two closest $B$ ions and all oxygens bonded to these $B$ ions was sufficient. In all cases, we found only positive frequencies for the optimized configurations while the transition states each had exactly one imaginary frequency corresponding to the reaction coordinate.

In order to compare the proton transport rate through the membrane materials, we need to determine the protonic concentration and diffusion rate constant. Here, the protonic concentration, $\left[\mathrm{OH}_{\mathrm{O}}\right]$, (charge symbol is omitted for clarity) is calculated by assuming equilibrium with gaseous hydrogen $\left(p_{\mathrm{H}_{2}}=1\right.$ bar) while enforcing site conservation and electroneutrality. From these standard assumptions, we obtain ${ }^{36}$
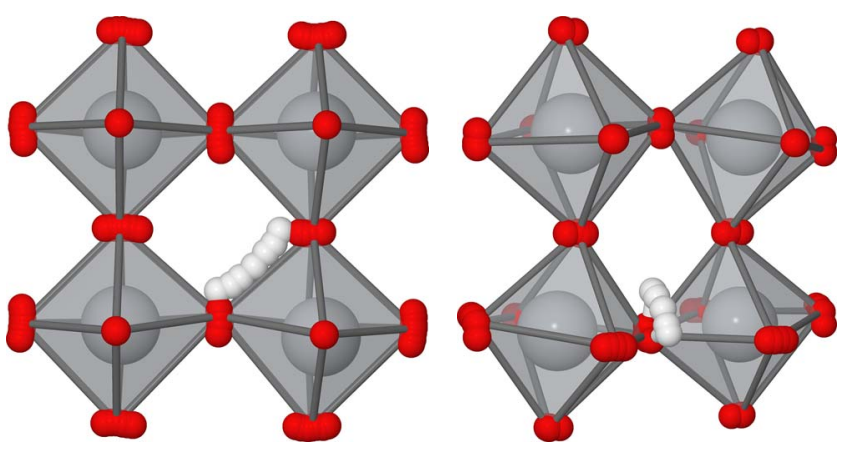

FIG. 3. (Color online) Illustration of conformational changes in $\mathrm{SrTiO}_{3}$ during (a) "H jump" and (b) "OH rot" diffusional processes. Octahedra illustrate the transition state structure. [Sr: omitted for clarity, Ti: gray (large), O: red, and $\mathrm{H}$ : white].

$$
\left[\mathrm{OH}_{\mathrm{O}}\right]=\frac{\mathrm{K}(T)}{2}\left(\sqrt{1+\frac{4}{\mathrm{~K}(T) V}}-1\right),
$$

where $\mathrm{K}(T)=\exp \left(-\Delta G / k_{B} T\right)$ is the equilibrium constant of reaction (2). $V$ is the unit-cell volume and $\Delta G=\Delta E_{\mathrm{OH}}$ form $-T \Delta S$ is the change in Gibbs free energy. The entropy of the gaseous hydrogen is determined by assuming a constant heat capacity in the temperature range of interest (500-1500 K) while the entropy of the absorbed hydrogen atom can be determined by considering the $\mathrm{OH}$ pairs as independent harmonic oscillators.

An effective rate constant for the two-step transport defined as

$$
k_{e f f}(T)=\left[k_{\mathrm{OH} \text { rot }}^{-1}(T)+k_{\mathrm{H} \text { jump }}^{-1}(T)\right]^{-1}
$$

provides a simple way of comparing diffusional properties of the different compounds. $k_{e f f}(T)$ from Eq. (4) is thus an upper bound to the true rate constants. It should be emphasized that the absolute rates obtained here are not expected to be directly comparable to experimentally obtained data due to

TABLE II. Energy barriers and pre-exponential factors for the two fundamental Grotthuss-type diffusion processes ( $\mathrm{H}$ jump and $\mathrm{OH}$ rot) calculated by the NEB method.

\begin{tabular}{lccccc}
\hline \hline & \multicolumn{2}{c}{ Barrier $(\mathrm{eV})$} & & \multicolumn{2}{c}{ Pre-exponential $\left(\mathrm{s}^{-1}\right)$} \\
\cline { 2 - 3 } \cline { 5 - 6 } Perovskite & H jump & OH rot & & $A_{\mathrm{H} \text { jump }}$ & $A_{\text {OH rot }}$ \\
\hline $\mathrm{BaTiO}_{3}$ & 0.18 & 0.16 & & $1.6 \times 10^{13}$ & $1.7 \times 10^{13}$ \\
$\mathrm{BaZrO}_{3}$ & 0.27 & 0.10 & & $8.9 \times 10^{12}$ & $9.6 \times 10^{12}$ \\
$\mathrm{BaNbO}_{3}$ & 0.12 & 0.12 & & $1.4 \times 10^{13}$ & $1.2 \times 10^{13}$ \\
$\mathrm{CaMnO}_{3}$ & 1.76 & 0.32 & & $1.2 \times 10^{15}$ & $1.4 \times 10^{13}$ \\
$\mathrm{CaTiO}_{3}$ & 1.68 & 0.28 & & $1.2 \times 10^{13}$ & $6.1 \times 10^{14}$ \\
$\mathrm{CaZrO}_{3}$ & 2.51 & 0.47 & & $4.9 \times 10^{14}$ & $3.2 \times 10^{13}$ \\
$\mathrm{PbTiO}_{3}$ & 0.30 & 0.13 & & $1.2 \times 10^{14}$ & $8.6 \times 10^{12}$ \\
$\mathrm{PbZrO}_{3}$ & 0.34 & 0.13 & & $1.1 \times 10^{13}$ & $4.0 \times 10^{13}$ \\
$\mathrm{SrNbO}_{3}$ & 0.26 & 0.11 & & $1.3 \times 10^{13}$ & $2.8 \times 10^{13}$ \\
$\mathrm{SrTiO}_{3}$ & 0.33 & 0.16 & & $8.0 \times 10^{13}$ & $7.9 \times 10^{12}$ \\
$\mathrm{SrZrO}_{3}$ & 0.80 & 0.22 & & $3.9 \times 10^{13}$ & $1.2 \times 10^{13}$ \\
\hline \hline
\end{tabular}






FIG. 4. (Color online) Proton transport frequency, $\Gamma$, as a function of temperature.

the undoped and defect free nature of this model.

We note that most experimentally measurable properties such as flux, diffusivity, and resistivity scale linearly with the protonic concentration and approximately linear with the effective rate constant from Eq. (4). ${ }^{30}$ Here we introduce the "proton transport frequency" as the product of these

$$
\Gamma(T)=k_{e f f}(T)\left[\mathrm{OH}_{\mathrm{O}}\right]
$$

which is proportional to the hydrogen flux. The proton transport frequencies are illustrated as a function of temperature in Fig. 4.

By initially comparing the activation energies of the two processes, we found a strong correlation. The two diffusional steps seemed hindered or favored by an underlying property of the material. We found a correlation of similar high quality between the diffusional barriers and $\Delta E_{\mathrm{OH} \text { form }}$; the freeenergy difference of reaction (2). See Fig. 5. It is clear that a stable protonic defect implies large activation barriers for both diffusional steps.

This inverse proportionality between mobility and concentration has been found in several other studies and is an important point to emphasize. The task of optimizing the overall protonic flux through the membrane is therefore a task of finding the optimal tradeoff between mobility and concentration. This is true for both pure and doped materials. It is known that $A$-site doping can increase the protonic con-
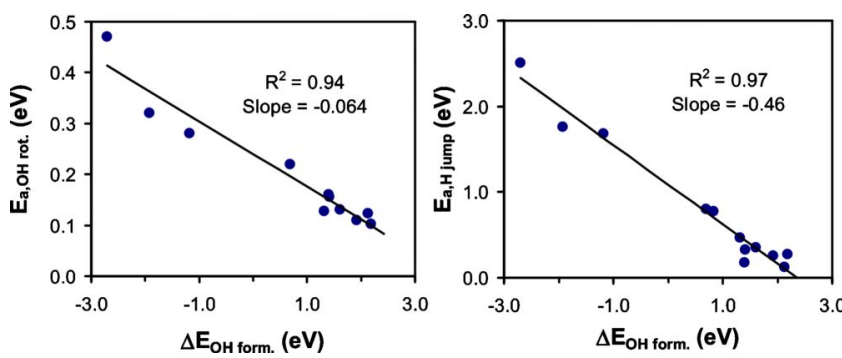

FIG. 5. (Color online) Correlations between $E_{a, \mathrm{OH} \text { rot }}, E_{a, \mathrm{H} \text { jump}}$, and $\Delta E_{\mathrm{OH}}$ form.

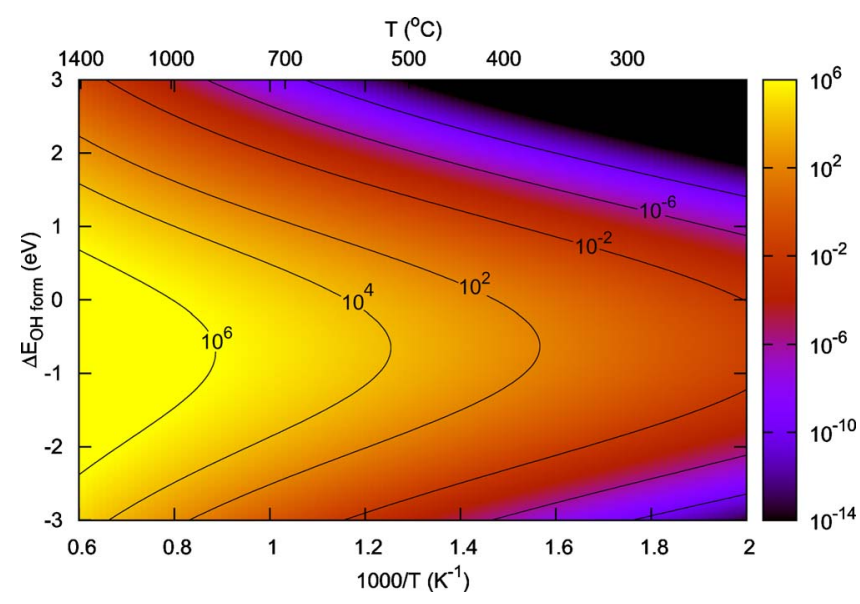

FIG. 6. (Color online) The proton transport frequency, $\Gamma$, in mol cm$~_{-3} \mathrm{~s}^{-1}$ as a function of temperature and $\Delta E_{\mathrm{OH}}$ form.

centration dramatically ${ }^{2,37}$ but dopants can also have a trapping effect on the protons and thereby lowering the mobility. ${ }^{38}$

The correlation scaling factor is quite different for the two Grotthuss processes investigated. While the $\mathrm{OH}$ rot barriers depend weakly on $\Delta E_{\mathrm{OH} \text { form }}$, the $\mathrm{H}$ jump barriers are strongly dependent with a ratio of approximately $0.5 \mathrm{eV}$ barrier per $\mathrm{eV} \Delta E_{\mathrm{OH}}$ form apparent from the slopes in Fig. 5. This can be explained by the difference in the dynamics of the processes. In the rotational process, the proton stays closely bound to the oxygen and remains "shielded" by the oxygen. It is thus expected that this activation barrier should be less sensitive to the composition of the material. The opposite can be stated for the $\mathrm{H}$ jump process. The proton has to leave the parent oxygen by breaking the $\mathrm{OH}$ bond from where much of the stabilization of the protonic defect stems.

It is interesting that the correlation is between these properties since it then is possible to determine the diffusion "flux" via just the thermodynamics of reaction (2), with only $T$ and $\Delta E_{\mathrm{OH}}$ form as variables, via the relations

$$
\begin{gathered}
E_{a, \mathrm{OH} \text { rot }}=-0.064 \Delta E_{\mathrm{OH} \text { form }}+0.24 \mathrm{eV}, \\
E_{a, \mathrm{H} \text { jump }}=-0.46 \Delta E_{\mathrm{OH} \text { form }}+1.09 \mathrm{eV}
\end{gathered}
$$

apparent from Fig. 5. Hereby it is possible to estimate $\Gamma$ as a volcano curve as a function of $\Delta E_{\mathrm{OH}}$ form and temperature with good accuracy. See Fig. 6 .

We see that the maximum flux, for a pure and undoped material, will be found at varying values of $\Delta E_{\mathrm{OH}}$ form dependent on temperature, as a result of entropy. At temperatures relevant for hydrogen permeable membranes $\left(300-800{ }^{\circ} \mathrm{C}\right)$, the maximum is found around $\Delta \mathrm{E}_{\mathrm{OH}}$ form $=-0.5 \mathrm{eV}$ and it is noticeable that none of the materials investigated are found in this desirable region. Even the closest materials, $\mathrm{SrZrO}_{3}$ and $\mathrm{CaTiO}_{3}$ with $\Delta E_{\mathrm{OH}}$ form of 0.69 and $-1.32 \mathrm{eV}$, respectively, are far from possessing this optimal tradeoff between mobility and concentration. As can be seen from the logarithmic scale of Fig. 6, even a slight improvement in $\Delta E_{\mathrm{OH}}$ form could increase the flux significantly. 


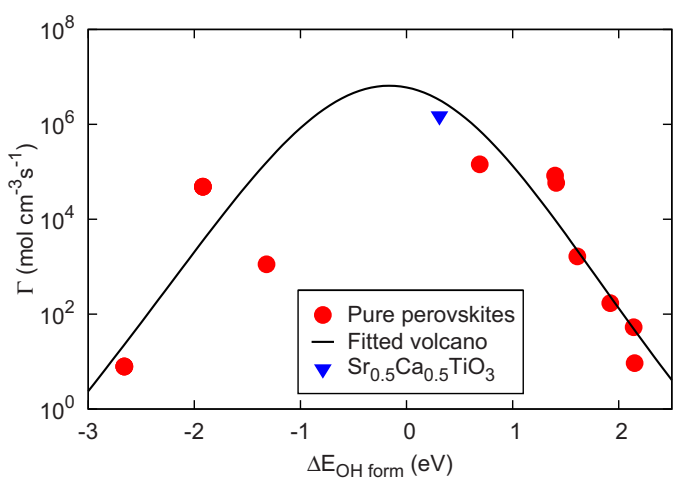

FIG. 7. (Color online) The proton transport frequency, $\Gamma$, in mol cm $\mathrm{cm}^{-3} \mathrm{~s}^{-1}$ as a function of $\Delta E_{\mathrm{OH}}$ form at $800{ }^{\circ} \mathrm{C}$. The alloyed perovskite has more than one order of magnitude higher protonic flux than any of the pure perovskites. The compositions of the pure perovskites are listed in Table I.

A method for designing membranes based on this seem possible since we have demonstrated that the binding energy and flux are correlated. Starting from a given material, e.g., $\mathrm{CaTiO}_{3}$, we now know that the main underlying problem is that the proton binds too strongly to the lattice oxygen $\left(\Delta E_{\mathrm{OH}}\right.$ form $\left.=-1.32 \mathrm{eV}\right)$, leading to too low a protonic mobility, which is not sufficiently compensated by the increased protonic concentration. We also know that, e.g., $\mathrm{SrTiO}_{3}$ has the opposite properties $\left(\Delta E_{\mathrm{OH}}\right.$ form $\left.=1.40 \mathrm{eV}\right)$. It thus seems possible that an alloy of these materials might have the desired thermodynamic properties leading to an increased overall diffusion rate.

To test this hypothesis, we set up a cubic $(2 \times 2 \times 2)$ $\mathrm{Sr}_{0.5} \mathrm{Ca}_{0.5} \mathrm{TiO}_{3}$ supercell as for the undoped compounds. The optimized cell constant of $(3.908 \AA)^{3}$ differ approximately $3 \%$ from the experimentally found value. ${ }^{16}$ Even though $\mathrm{Sr}_{0.5} \mathrm{Ca}_{0.5} \mathrm{TiO}_{3}$ is structurally more complicated than a pure perovskite we note that all oxygens and $\mathrm{OH}$ bonds are equivalent when the material is assumed fully mixed. The formation energy of the protonic defect was hereafter determined to $0.31 \mathrm{eV}$ comparable to the average value of the two pure compounds (approximately $0 \mathrm{eV}$ ). When determining the kinetic barriers we note that, as for the pure compounds, only one proton jump is found but two different $\mathrm{OH}$ rotations must be taken into consideration. Namely, one rotation past a $\mathrm{Sr}$ ion and another rotation past a $\mathrm{Ca}$ ion both necessary for diffusion through an entire unit cell. The energy barriers of these three processes were determined to $0.26 \mathrm{eV}, 0.32 \mathrm{eV}$, and $0.30 \mathrm{eV}$ with exponential prefactors of $5.2 \times 10^{12} \mathrm{~s}^{-1}$, $1.9 \times 10^{13} \mathrm{~s}^{-1}$, and $2.5 \times 10^{13} \mathrm{~s}^{-1}$, respectively. The proton transport frequency for $\mathrm{Sr}_{0.5} \mathrm{Ca}_{0.5} \mathrm{TiO}_{3}$ is plotted in Fig. 7 and we see that the correlations seem to hold for this compound as well. It is noteworthy that this mixed titanate is predicted to have considerably higher proton flux than any of the pure materials in the temperature range of interest-even the zirconates. The exact position of the top of the volcano is however sensitive to the limited number of data points on the left-hand side of Fig. 7 but the material is potentially very promising.

A similar approach to designing materials has already successfully been applied for several metallic catalysts ${ }^{39}$ and since experimentally determining the best level of doping can be a highly time consuming and expensive task such correlations can help to guide the experimentalist when selecting dopants and dopant concentrations. This study reveal that it may be possible to predict optimal doping levels using correlation schemes determined by DFT.

Correlations between structural parameters, e.g., ionic radii and kinetic or thermodynamic properties are highly desirable since they could help to design materials from even simpler and more readily available data. Correlations with structural parameters of the same quality as the correlations described so far were not found but we did however find single logarithmic correlations of decent quality, $R^{2}=0.79$ and 0.60 , between the ionic radius of the $A$ ion and the barriers for $\mathrm{H}$ jump. and $\mathrm{OH}$ rot., respectively. In both cases, we find that generally the larger $A$ ion yield smaller barriers, consistent with the findings by Mitsui et al. ${ }^{5}$ but this was not explored further.

\section{CONCLUSIONS}

We have investigated hydrogen/proton dynamics in a series of undoped perovskites using DFT. The preferred site for protonic defects and the reaction paths in the Grotthuss-type diffusion process have been determined. Transition states, energy barriers, and proton transport frequencies have been determined. We find a strong correlation between the barriers of diffusion and the binding energy of the proton indicating that the stronger the proton binds to the oxygen, the higher is the energy barrier for diffusion.

We thus find that the proton concentration and proton mobility are correlated properties and we deduce a direct relation between the proton transport and the binding energy. This correlation is useful for screening candidate membrane materials without performing expensive reaction path calculations; only calculating the binding energy, $\Delta E_{\mathrm{OH}}$ form. We find no pure perovskites possessing the optimum thermodynamics and by this we also stress the importance of acceptor doping. Finally, we demonstrate the possibility of predicting membrane materials with better tradeoffs between mobility and concentration of protons in the membrane by utilizing the correlations found.

\section{ACKNOWLEDGMENTS}

The authors would like to acknowledge the Danish Center for Scientific Computing, the Nordic Center of Excellence on Hydrogen Storage Materials, the Catalysis for Sustainable Energy (CASE) initiative and the Center of Atomic-Scale Materials Design (CAMD). CASE is funded by the Danish Ministry of Science, Technology and Innovation, and CAMD is funded by the Lundbeck foundation. 
*teve@ risoe.dtu.dk

${ }^{1}$ Y. Nagahara, S. Sugawara, and K. Shinohara, J. Power Sources 182, 422 (2008).

${ }^{2}$ K. D. Kreuer, Annu. Rev. Mater. Res. 33, 333 (2003).

${ }^{3}$ J. S. Hummelshøj et al., J. Chem. Phys. 131, 014101 (2009).

${ }^{4}$ J. Ranløv, N. Bonanos, F. W. Poulsen, and M. Mogensen, Diffus. Defect Data, Pt. B 39-40, 219 (1994).

${ }^{5}$ A. Mitsui, M. Miyayama, and H. Yanagida, Solid State Ionics 22, 213 (1987).

${ }^{6}$ A. Andreasen, T. Vegge, and A. S. Pedersen, J. Phys. Chem. B 109, 3340 (2005).

${ }^{7}$ K. D. Kreuer, Solid State Ionics 125, 285 (1999).

${ }^{8}$ All formal oxidation states are $2+$ and $4+$ and coordination numbers are 12 and 6 for the $A$ and $B$ metallic cations, respectively.

${ }^{9}$ I. Ahmed, S. G. Eriksson, E. Ahlberg, C. S. Knee, M. Karlsson, A. Matic, D. Engberg, and L. Boerjesson, Solid State Ionics 177, 2357 (2006).

${ }^{10}$ Y. Grin, H. Mueller-Buschbaum, and H. G. von Schnering, Z. Naturforsch. Teil B 52, 153 (1997).

${ }^{11}$ Q. Zhou and B. J. Kennedy, J. Phys. Chem. Solids 67, 1595 (2006).

${ }^{12}$ A. T. Zayak, X. Huang, J. B. Neaton, and K. M. Rabe, Phys. Rev. B 74, 094104 (2006).

${ }^{13}$ I. Levin, T. G. Amos, S. M. Bell, L. Farber, T. A. Vanderah, R. S. Roth, and B. H. Toby, J. Solid State Chem. 175, 170 (2003).

${ }^{14}$ Y. Kuroiwa, Y. Terado, Kim Su Jae, A. Sawada, Y. Yamamura, S. Aoyagi, E. Nishibori, M. Sakata, and M. Takata, Jpn. J. Appl. Phys., Part 1 44, 7151 (2005).

${ }^{15}$ H. Hannerz, G. Svensson, S. Ya. Istomin, and O. G. D'yachenko, J. Solid State Chem. 147, 421 (1999).

${ }^{16}$ T. Yamanaka, N. Hirai, and Y. Komatsu, Am. Mineral. 87, 1183 (2002).

${ }^{17}$ B. J. Kennedy, C. J. Howard, A. K. Prodjosantoso, and B. C. Chakoumakos, Appl. Phys. A 74, S1660 (2002).

${ }^{18}$ M. Hikam, B. Soegijono, and N. I. Sofyan, J. Appl. Sci. 6, 3096 (2006).

${ }^{19}$ K. D. Kreuer, Chem. Mater. 8, 610 (1996).
${ }^{20}$ T. Norby and P. Kofstad, Solid State Ionics 20, 169 (1986).

${ }^{21}$ H. K. Bentzer, N. Bonanos, and J. W. Phair, Solid State Ionics 181, 249 (2010).

${ }^{22}$ M. Karlsson, A. Matic, D. Engberg, M. E. Björketun, M. M. Koza, I. Ahmed, G. Wahnström, L. Börjesson, and S. G. Eriksson, Solid State Ionics 180, 22 (2009).

${ }^{23}$ J. Voss, Q. Shi, H. S. Jacobsen, M. Zamponi, K. Lefmann, and T. Vegge, J. Phys. Chem. B 111, 3886 (2007).

${ }^{24}$ DACAPO pseudopotential code, http://www.fysik.dtu.dk/campos/ dacapo

${ }^{25}$ J. P. Perdew and Y. Wang, Phys. Rev. B 45, 13244 (1992).

${ }^{26}$ D. Vanderbilt, Phys. Rev. B 41, 7892 (1990).

${ }^{27}$ H. J. Monkhorst and J. D. Pack, Phys. Rev. B 13, 5188 (1976).

${ }^{28}$ G. Mills, H. Jónsson, and G. Schenter, Surf. Sci. 324, 305 (1995).

${ }^{29}$ H. Jónsson, G. Mills, and K. W. Jacobsen, Classical and Quantum Dynamics in Condensed Phase Simulations (World Scientific, Singapore, 1998).

${ }^{30}$ P. Hänggi, P. Talkner, and M. Borkovec, Rev. Mod. Phys. 62, 251 (1990).

${ }^{31}$ T. Vegge and K. W. Jacobsen, J. Phys. Condens. Matter 14, 2929 (2002).

${ }^{32}$ T. Vegge, Phys. Rev. B 70, 035412 (2004).

${ }^{33}$ R. F. W. Bader, Can. J. Chem. 76, 973 (1998).

${ }^{34}$ N. Bork, N. Bonanos, J. Rossmeisl, and T. Vegge (unpublished).

${ }^{35}$ M. Björketun, Ph.D. thesis, Chalmers University of Technology, 2007.

${ }^{36}$ P. Kostad, Non-Stoichiometry, Diffusion and Electrical Conductivity in Binary Metal Oxides (Wiley-Interscience, New York, 1972).

${ }^{37}$ T. Norby, M. Viderøe, R. Glöckner, and Y. Larring, Dalton Trans. 2004, 3012.

${ }^{38}$ M. E. Björketun, P. G. Sundell, and G. Wahnström, Phys. Rev. B 76, 054307 (2007).

${ }^{39}$ J. K. Nørskov, T. Bligaard, B. Hvolbæk, F. Abild-Pedersen, I. Chorkendorff, and C. H. Christensen, Chem. Soc. Rev. 37, 2163 (2008). 